GaAs, indicating the changing balance from predominantly covalent to predominantly ionic character.

The ionicity parameter of Phillips and Van Vechten is a function of various energy levels in the crystal, and varies from zero to unity in going from fully covalent to fully ionic bonding. Phillips (Rev. Mod. Phys., 42, 317; 1970) noticed that for sixty-eight binary crystals, a value of 0.785 neatly separated the more covalent four-fold coordinated crystals of the structure of wurzite or zinc blende from the more ionic crystals of the rocksalt structure. This value was arrived at without any theoretical justification for a sharp division. Walter and Cohen have surmised that the change of crystal structure comes about as the covalent bonding vanishes, the limits of the bonding charge being defined from the position of the outermost closed contour. They have therefore plotted values for this charge against ionicity for two series of crystals, Ge, GaAs and $\mathrm{ZnSe}$ in the fourth period of the periodic table and $\alpha$-Sn, InSb and CdTe in the fifth period. The values for the ionicity at zero bonding charge were 0.79 and 0.78 respectively, in excellent agreement with Phillips's figure. Thus the notion of a well defined ionicity as the threshold between structures of different coordination and bonding can be seen to have some basis in the model of pseudopotential band theory.

\section{GEYSERS \\ Man-made Eruptions}

from our Geomagnetism Correspondent

So many Earth scientists today work with a perpetual image of seafloor spreading at the backs of their minds that it is good to be reminded, once in a while, that there are other things in geophysical life besides the new global tectonics. Take geysers, for instance: the 1,728-page Dictionary of Geophysics apparently contains not one reference to it. Nobody imagines, of course, that the study of geyser action is going to bring about any sort of revolution in the Earth sciences; but it would be a great pity if this and other lesser-known phenomena were to receive no attention at all. Fortunately, this is not the case. J. S. Rinehart, for example, has been investigating the thermal and seismic properties of geysers for many years, having made a special study of America's most famous example, the Old Faithful geyser in Yellowstone National Park.

Rinehart (J. Geophys. Res., 75, 6714; 1970) has now turned his attention to an artificial geyser, Crump well, in Lake County, Oregon. It may come as something of a revelation to learn that there is such a thing as a man-made geyser at all. The $15 \mathrm{~cm}$ diameter Crump well was drilled, however, to a depth of $35 \mathrm{~m}$ fifteen years ago in an unsuccessful attempt to develop geothermal power in a known geothermal area. The upper $17.7 \mathrm{~m}$ of the hole is in alluvial fill below which lies $2.1 \mathrm{~m}$ of water-bearing sand. The rest of the hole is drilled into solid lava. The interesting thing about this otherwise undistinguished well is, however, that every $8-10 \mathrm{~h}$ it erupts to a height of $30-40 \mathrm{~m}$ and continues to erupt for about a minute each time. Each chief eruption is then followed a few minutes later by a second, lesser outburst, and occasionally by a third.

Why does this well erupt at all ? From a series of temperature profiles down the well at various times throughout the eruption cycle, Rinehart has been able to piece together the events leading to an eruption. An hour or so after the previous eruption, the temperature at every point in the well lies below the boiling point curve, but not everywhere by an equal interval. At about $7.5 \mathrm{~m}$ from the top the temperature is less than $1^{\circ} \mathrm{C}$ from boiling; but in other places the temperature difference may be anything up to $30-40^{\circ} \mathrm{C}$. As time goes on, the water at the $7.5 \mathrm{~m}$ depth boils. Steam bubbles rise in the well but are quickly condensed by the cooler water above, which is warmed as a result. Because of the flashing of water into steam at the $7.5 \mathrm{~m}$ level the temperature there is lowered, but soon rises again to produce another flash. In other words, at this point the temperature periodically rises above and falls below the boiling point. While all this is going on, the water in the upper $7.5 \mathrm{~m}$ circulates in the pipe but the water below remains steady.

As the process proceeds, the water in the lower part of the pipe begins to heat up. Immediately before an eruption there is a second temperature peak at about 18-19 m, which would seem to indicate that heat is being supplied through the thin porous sand layer possibly in the form of hot water. Finally, there comes a point when the water above $7.5 \mathrm{~m}$ is no longer able to absorb the heat from the rising bubbles. The steam then suddenly blows off the head of superheated water. This is the observed eruption.

Why there should be a second eruption soon after the first is not quite clear. Rinehart found it impossible to measure the temperature profile between the two eruptions because of the short time interval involved. But what probably happens is that the hot water from below which replaces the ejected water enters a preheated pipe and rapidly reaches the unstable situation.

\title{
Problem of Excess X-rays Eased
}

HARD on the heels of the MIT survey of the X-ray sources in the direction of the galactic centre (Nature, 229, 96; 1971), Pounds's group at Leicester is this week publishing its survey of an adjacent area of sky between galactic latitudes $310^{\circ}$ and $220^{\circ}$. The Leicester group is making a speciality of this region, which corresponds to the southern hemisphere constellations Norma, Lupus and Centaurus. The group's latest article -in next Monday's Nature Physical Science-reports data from a Skylark rocket flight in April 1969, and comparison with a similar experiment a year earlier (reported by Cooke, Griffiths and Pounds, Nature, 224, 134; 1969) shows that some of the sources can be counted among the $\mathrm{X}$-ray variables. This is the classification that was opened with the appearance in 1967 and subsequent decay of Cen X-2, which the second Leicester flight shows to be still faintly detectable in spite of previous evidence to the contrary. As well as Cen X-2, the sources Cen $X-3$ and Lup X-1 seem to be variable according to the comparison and were more intense during the second flight.

Altogether seven sources are covered in the survey which was sensitive to energies between 1 and $12 \mathrm{keV}$, and including those mentioned already the sources whose strengths are given are Nor X-1 and X-2 and Cen X-1 and X-5. Cen $X-5$ is a new source, not recognized before, which is below the sensitivity levels of earlier surveys.

The Leicester group has been particularly concerned with a background flux of X-rays coming from the plane of the galaxy which on the face of it is not attributable to identifiable point sources. Such an excess of X-radiation, coming from the Centaurus-Carina-Vela area, was recognized in the 1968 flight and several mechanisms were put forward-suprathermal proton bremsstrahlung, electron capture from neutral hydrogen by heavy nuclei in cosmic rays, inverse Compton scattering of starlight or the blackbody radiation, or simply unresolved sources along the galactic equator that are below the sensitivity threshold.

With the data from the second Leicester flight to play with, the situation now looks much simpler. When the sources Cen $X-1, X-2, X-3$ and $X-5$ that appear in the data from the second flight but which were not unambiguously resolved in 1968 are allowed for in the 1968 data, only ten per cent of the excess background flux is left unaccounted for, in the Centaurus region at least. 